\title{
Mots et mémoires : Vocabulaire de l'internement des japonais améri- cains pendant la Deuxième Guerre mondiale
}

\begin{abstract}
En février 1942, le Président américain Franklin D. Roosevelt signe l'ordre exécutif 9066. Cet ordre permet au Secrétaire d'État à la Guerre de qualifier des zones aux États-Unis comme zones

L'auteur : Persephone HERnANDEZ-Vogt est titulaire d'un master en science politique.

militaires, d'où peuvent être exclues certaines personnes. Roosevelt ne spécifie pas l'ethnie des personnes exclues, laissant le choix au Secrétaire d'État, mais en pratique, cette décision a comme effet de mettre dans des camps plus de 120.000 personnes d'origine ou de descendance japonaise et quelque 1.000 personnes allemandes et italiennes'. Roosevelt considère que l'ordre est nécessaire pour protéger les États-Unis de l'espionnage et du sabotage, mais les générations futures ne verront pas cette action comme nécessaire. Au contraire, cet acte jettera une ombre sur l'histoire américaine.
\end{abstract}

\section{Le choix des mots}

Travailler sur ce sujet oblige une évaluation des termes utilisés pour décrire les camps dans lesquels se trouvaient les Japonais américains. II existe déjà un grand débat sur cette terminologie, et ce débat est compliqué par l'existence de plusieurs types de camps. Le gouvernement américain parlait de trois types de camps: les assembly centers, les relocation camps ou relocation centers et les internment camps $^{2}$. Cette question est importante pour l'étude de la mémoire parce qu'elle détermine la façon dont la génération des camps se souvient de ses expériences et dont les générations futures en parlent. La diversité des camps rend difficile de rassembler sous un même vocable les expériences de ceux qui furent enfermés. Le choix d'un terme est donc essentiel à la création des mémoires.

'Okamura Raymond, "The American Concentration Camps: A Cover-Up Through Euphemistic Terminology ", The Journal of Ethnic Studies, 1982, vol. 10, n³, pp. 95-109.

${ }^{2}$ Kotek Joël et Rigoulot Pierre, Le siècle des camps, Paris, JC Lattès, 2000, pp. 477-480 
Même s'il est difficile de dire quels sont les mots corrects, certains mots sont clairement incorrects. Raymond Okamura, dans son article The American Concentration Camps: A Cover-Up Through Euphemistic Terminology, examine l'usage de termes euphémistiques comme "l'évacuation " et "camps de relogement " par le gouvernement américain et le compare aux euphémismes utilisés par les nazis. II trouve non seulement une ressemblance mais aussi certaines nomenclatures identiques, telle que l'évacuation. La majeure partie de l'article d'Okamura se focalise sur l'usage de ces termes à l'époque même des camps, mais il critique aussi l'usage continu de ces mots actuellement (l'article fut écrit en 1982) : "The record needs rewriting $»^{3}$. Pour Okamura, les seuls termes valables sont soit « camp d'internement, " soit " camp de concentration ». James A. Hirabayashi, auteur d'un article intitulé 'Concentration Camp' or 'Relocation Center': What's in a Name? qui se base sur le texte d'Okamura, estime que seul le "camp de concentration" suffit, car les camps d'internement étaient réservés aux prisonniers politiques.

Le texte d'Okamura est lié aux questions de mémoire car il suggère fortement que le fait de parler de camps d'évacuation est une forme de négationnisme:

Since Japanese Americans were in fact confined against their will, the 'evacuation-relocation' nomenclature is a distortion. Those writing seem unable to accept the very fact that over 120,000 men, women, children, and babies were expelled from their homes and locked up in American concentration camps ${ }^{4}$.

Hirabayashi fait écho à l'écrit d'Okamura, à propos des euphémismes actuels, en en soulignant l'aspect négatif :

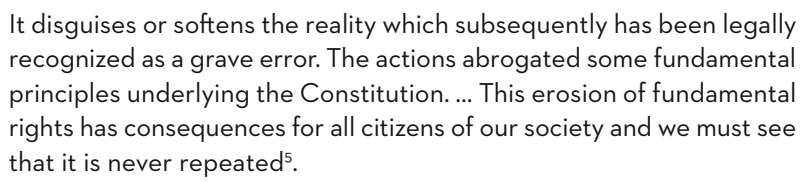

Il trouve que l'utilisation de la terminologie d'évacuation et de relogement stérilise l'histoire et minimise les crimes commis contre les Japonais et les Japonais américains. Parler de ces camps comme de camps d'évacuation laisse sous-entendre que les victimes furent déplacées pour les protéger et qu'elles ne furent pas forcées à entrer dans

${ }^{3}$ Okamura Raymond, "The American Concentration Camps: A Cover-Up Through Euphemistic Terminology ", op. cit., p. 105

${ }^{4} \mathrm{lbid}$., p. 95

${ }^{5}$ HiRABayAshi James, “ 'Concentration Camp' or 'Relocation Center' - What's in a Name? ", Enduring Communities, 2004, disponible à l'adresse suivante : http://www.discovernikkei.org/ (consultée le 9 novembre 2013). 
ces camps, alors que la vérité est tout autre : bien que le plupart des Japonais américains se sont volontairement rendus aux lieux déclarés, Okamura souligne qu'ils ne s'attendaient pas aux conditions des camps. Il cite le souvenir d'un survivant: "The evacuation did not seem too unfair until we got right to the camp and were met by soldiers with guns and bayonets " ${ }^{6}$. Les victimes de la soi-disant "évacuation" ne savaient pas ce qui les attendait. Comme dit Okamura : "Unless one took a great deal of trouble to find out, a general newspaper reader would not have known that native born Americans were being held prisoner in these camps ${ }^{7}$. De plus, la plupart des articles écrits au sujet des camps laissaient sous-entendre que les gens dans les camps étaient libres de partir. Le manque d'informations dans les journaux a certainement influencé le développement du négationnisme car les Américains qui ne furent pas directement affectés par l'évènement n'ont pas de souvenirs et peuvent ne pas faire face aux actes de leur pays.

Si l'idée de "camps d'évacuation " est trop euphémistique, le " camp de concentration " est-il le seul terme possible? Comme nous avions vu, Hirabayashi répond par l'affirmative. Il est vrai que les camps aux États-Unis durant la Deuxième Guerre Mondiale ont les caractéristiques d'un camp de concentration si on s'en réfère à Hirabayashi citant la définition de Webster au début de son article : "places where political prisoners are placed under armed guards " ${ }^{8}$. De leur côté, Kotek et Rigoulot, dans leur livre Le siècle des camps, s'interrogent également :

S'agit-il à proprement parler de camps de concentration? Oui, dans la mesure où leur fonction première est de rassembler en un lieu isolé et ceint de barbelés des individus considérés comme des menaces pour la société et dont on veut protéger le reste du corps social. Oui encore, dans la mesure où les individus qui y sont internés, le sont sans avoir fait l'objet d'aucune inculpation ni jugement préalables?.

Mais les auteurs soulignent que "les conditions qui y prévalent n'ont que très peu de rapport avec celles des camps de concentration des nazis ou soviétiques. II n'est pas question ici d'éliminer des êtres humains par le travail ou les mauvais traitements, mais de les isoler " $^{10}$. Ceci nous amène donc à questionner le terme " camp de concentration ". II a perdu sa définition objective. Comme le disent Kotek et

${ }^{\circ}$ Okamura Raymond, "The American Concentration Camps: A Cover-Up Through Euphemistic Terminology ", op. cit., p. 103

7 Ibid., pp. 102-103

${ }^{8}$ HIRABAYASHI James, « 'Concentration Camp' or 'Relocation Center' - What's in a Name? »,op. cit.

${ }^{9}$ KOTEK Joël et Rigoulot Pierre, Le siècle des camps, op. cit., p. 480

10 Ibid., p. 480 
Rigoulot, c'est un « mot malade» :

[ll est source] à l'instar de "génocide " ou d' "holocauste ", de bien de confusions et de bien trop de relativisme. Sources et signes, car l'idée qu'il existe dans des situations et des systèmes très différents des lieux susceptibles d'être tous désignés du nom de «camp de concentration " renvoie à l'aveuglement dont on a longtemps fait preuve en Occident à l'égard des camps du monde communiste. [...] Quoi qu'en disent certains - et pas seulement pour des raisons quantitatives - nous démontrerons que Auschwitz ne peut se comparer à Manzanar, un camp d'internement des Américains d'origine japonaise »".

C'est en fait la coïncidence entre l'internement des Japonais aux États-Unis et la mise en place des camps nazis qui crée des problèmes. Hirabayashi admet que des personnes juives peuvent s'offenser si on qualifie les camps de "concentration ", étant donné que les individus incarcérés dans les camps aux États-Unis ne furent pas tués de manière systématique, ni traités de la même façon ${ }^{12}$. Hirabayashi rejette cet argument et alimente, selon nous, la concurrence des victimes. Pour lui, les actions d'Hitler étaient légales sous les lois du Troisième Reich, alors que les actions du Gouvernement américain violaient la Constitution. Dans la mémoire collective, la Shoah revêt régulièrement l'image de la plus grande cruauté humaine. Hirabayashi, en considérant les actions du Gouvernement américain comme plus ignobles que celles des nazis, augmente ainsi la valeur de la souffrance des victimes japonaises américaines. De façon intentionnée ou non, ses mots tentent d'évoquer plus de sympathie pour l'expérience de ces victimes en mobilisant les images de la Shoah et en les appliquant aux camps américains. De ce fait, la Shoah entre en « concurrence " avec les camps aux États-Unis par sa simple existence : les deux événements ayant lieu en même temps, la mémoire collective des Américains qui ne sont pas d'origine ou de descendance japonaise, est dominée par la Shoah, car il est plus facile d'accepter une histoire où son pays agit de manière héroïque que de faire face à une histoire où son pays est coupable ${ }^{13}$.

\footnotetext{
" Kotek Joël et RIgoulot Pierre, Le siècle des camps, op. cit., p. 19

${ }^{12}$ Cela dit, comme l'écrivent Kotek et Rigoulot, les conditions hygiéniques nétaient pas très bonnes, ce qui entrainait la mort. De plus, il y avait des gardes armés dans la plupart des camps pour tirer sur ceux qui essayaient de s'échapper

${ }^{13}$ "Remettre en avant le « roman national "? Lequel ? Au lieu d'intégrer les micro-romans des minorités au roman national revu et corrigé, le pouvoir veut en revenir au vieux récit des manuels de Lavisse vidés au demeurant de leurs valeurs républicaines " (ROBIN Régine, "La France et la concurrence des mémoires: l'impossibilité d'assumer le passé ", in GRANDJEAN Geoffrey et JAMIN Jérôme (dir.), La Concurrence mémorielle, Paris, Armand Colin, 2011, pp. 23-37, 35 p.), dit Régine Robin au sujet de la France ; il s'agit d'un phénomène pareil aux Etats-Unis, sauf que ce n'est pas le gouvernement mais le peuple qui prend l'initiative.
} 
Pour résoudre le problème de vocabulaire posé par les camps, l'idée de sous rature avec ses origines dans le travail de Martin Heidegger, mais modifié par Jacques Derrida, peut nous être utile. Quand un mot est inexact mais nécessaire, ces deux penseurs le mettent sous rature. Selon Derrida, le problème n'est pas avec les mots individuels mais avec le système linguistique dans lequel ils apparaissent, qui est imprécis et contorsionné. On doit barrer le mot en question car il n'est pas tout à fait juste, mais il doit rester car les alternatives à ce mot posent autant de problèmes ${ }^{14}$. Derrida utilise cette technique pour éclairer le manque de "vraie " connexion entre le signifiant et le signifié ${ }^{15}$; l'être humain choisit comment représenter un objet sans avoir une raison de choisir un symbole plutôt qu'un autre. Pour notre part, nous suggérons d'appliquer cette idée d'une façon différente, à l'utilisation du terme " camps de concentration" pour décrire les camps aux États-Unis. Ici, la relation entre signifiant et signifié n'est pas implicite, comme pour tous les mots, mais il y a encore une couche : le signifiant évoque un autre signifié que celui signifié, par la force de l'association avec la Shoah. Si on utilise la "sous rature " pour illustrer cette problématique, on écrit : camps de eoncentration. Ainsi, le lecteur peut comprendre que les camps étaient de camps de concentration, mais la rature lui rappelle que ce mot est inadéquat.

Une possibilité un peu plus accessible au public est de les appeler des camps d'internement. Ce terme exprime que les personnes dans les camps étaient des prisonniers politiques et il donne aux expériences des Japonais américains son propre nom. Cela dit, cette classification n'évite pas le problème de la nature inadéquate des mots ; comme nous avons vu, les camps d'internement étaient une sous-catégorie des camps pour les Japonais américains, et le terme reste inexact. Cependant, un auteur qui a déjà reconnu que ces camps furent des camps de concentration peut alors envisager ces mots moins «malades ». Kotek et Rigoulot choisissent ce terme dans l'introduction de leur livre ${ }^{16}$. Leur définition d'un camp d'internement renvoie à un endroit qui sert à " isoler temporairement des individus suspects ou dangereux. Entrent dans cette catégorie les camps créés durant les conflits pour interner les nationaux 'ennemis', ou ceux perçus comme tels (par exemple, les Japonais aux États-Unis) $»^{17}$. En contraste, ils définissent les camps de concentration comme ayant 
"une quadruple logique d'avilissement, de rééductation, de travail et d'anéantissement $\aleph^{18}$. Ces camps se distinguent également des camps d'internement car ils sont créés avec l'intention d'une longue durée. Les deux camps se distinguent donc de par leur fonction et leur temporalité.

Une autre solution qui reconnaît cette complexité est d'alterner les termes. C'est ce que fait George Takei, un homme qui passa une partie de son enfance dans des camps, sur le site internet de sa pièce de théâtre musicale, Allegiance. Célèbre pour son rôle dans Star Trek (la série télévisée des années 1960), sa page facebook (qui a plus de 5 millions d'abonnés) et son activisme pour les droits des homosexuels, Takei utilise sa célébrité pour attirer le regard du public sur l'histoire de l'internement. Le site internet d'Allegiance contient une section informative sur le Japanese American Internment :

The internment of Japanese Americans during World War II is an oft-overlooked and shameful chapter of American history. In the aftermath of Japan's surprise attack on Pearl Harbor, President Roosevelt authorized, through Executive Order 9066, the forced evacuation of approximately 120,000 persons of Japanese descent from the West Coast of the U.S. and their involuntary internment in ten concentration camps on American soil. About two-thirds of the internees were American citizens ${ }^{19}$.

Comme on peut voir, Takei utilise les trois termes principaux : l'internement, les camps de concentration, et les évacuations (forcées). Cette solution incorpore toute l'histoire étymologique des camps.

Takei fait mention de la présence nombreuse des citoyens américains dans ces camps, une pratique présente aussi dans les textes d'Okamura, de Hirabayashi et de Kotek/Rigoulot. Le texte d'Okamura se concentre particulièrement sur la manière dont les documents de l'époque parlaient des citoyens dans les camps : "All written orders contained the curious phrase "non-alien, " which turns out to be a code word for a citizen of the United States of America ${ }^{20}$. La documentation des camps et les reportages des médias évitaient d'utiliser le mot «citoyen ». Cette façon de faire peut s'expliquer par le fait que la présence de citoyens dans les camps pouvait choquer le public. Aujourd'hui, au lieu de cacher la présence des citoyens, cette présence est soulignée pour la même raison. Le crime du Gouvernement

\footnotetext{
${ }^{18}$ Kotek Joël et Rigoulot Pierre, Le siècle des camps, op. cit., p. 21

19 TAKEl George, "Japanese American Internment ", Allegiance : A New American Musical, disponible à l'adresse suivante http://www.allegiancemusical.com/ (consultée le 14 novembre 2013) [souligné par l'auteure].

${ }^{20}$ Okamura Raymond, "The American Concentration Camps : A Cover-Up Through Euphemistic

Terminology ", op. cit., p. 99
} 
américain est vue très négativement par de nombreux individus quand il fut dirigé vers des citoyens, alors que l'incarcération des étrangers est davantage tolérée. Dans son article, "La Shoah, le génocide paradigmatique, " Kotek dit qu'un des éléments qui distingue la Shoah des autres génocides, est l' "innocence " des victimes, car ils sont non seulement non coupables mais aussi non nuisibles, dans le sens de "causer la mort »:

Or que dire sinon que les Herero, les Arméniens et les Tutsis furent, du point de vue de leurs bourreaux, objectivement dangereux, nuisibles et nocifs et ce, de par leur simple présence sur un territoire désormais convoité. Dans ces trois cas, le génocide peut être qualifié tout à la fois de pragmatique et de rétributif, pour couper court définitivement à la menace obsidionale ${ }^{21}$.

Selon Kotek, ceci donne un caractère exceptionnel à la Shoah. Les Japonais et les Japonais américains ne furent bien sûr pas victimes d'un génocide, mais cette théorie de nuisibilité permet de comprendre les propos de Kotek et Rigoulot quand ils se positionnent sur les analyses historiques de l'internement:

\begin{abstract}
Se refusant à toute mise en perspective, des historiens révisionnistes, au sens américain du terme s'entend - d'origine japonaise, ou non - s'en prennent vivement aux autorités américaines. Ces historiens semblent oublier que le Japon était effectivement en guerre avec les Etats-Unis et qu'il était l'allié de l'Allemagne nazie, pour ne s'intéresser qu'à l'aspect juridique de cet internement et à ses motivations cachées. ... La présence des sous-marins [japonais] ... est minimisée ${ }^{22}$.
\end{abstract}

Que les Japonais aux États-Unis soient «nuisibles » ou non du point de vue du Gouvernement américain, il n'en demeure pas moins que les Japonais américains, citoyens du pays qui les a internés, sont clairement non nuisibles puisque l'attaque doit être commise par un État extérieur et non par une groupe interne. C'est pour cette raison que plusieurs auteurs insistent sur la présence des citoyens dans les camps. A leurs yeux, l'élément le plus injuste de l'internement, est l'incarcération des Japonais alors qu'ils sont des citoyens comme les autres.

\title{
Les efforts de reconnaissance et les autorités américaines
}

Une des grandes organisations qui va se joindre aux mouvements pour les réparations est la Japanese American Citizens League (JACL). Créée bien avant la guerre, elle a milité en faveur de plusieurs textes 
légaux dans les années 1960 et 1970, celui de la rescision de l'ordre exécutif 9066 étant le plus intéressant pour cette étude ${ }^{23}$. C'est ainsi en utilisant des voies légales que les Japonais américains ont permis une reconnaissance officielle de leur passé. En 1976, l'activisme des Japonais américains et du JACL atteindra un de ses buts : la rescision de l'ordre exécutif 9066 par le Président Gerald Ford ${ }^{24}$. Cette décision de Ford n'est pas une loi mémorielle, même si, dans sa proclamation, le Président précise que l'ordre fut une erreur. D'une part, la branche exécutive est responsable. II ne s'agit donc pas d'un texte à valeur légale. D'autre part, c'est une correction d'un ordre qui n'aurait pas dû être donné. Cette rescision permet les réparations de 1988 sous le Civil Liberties Act, qui donne $\$ 20.000$ à chaque personne internée. Conscients qu'il crée un précédent, le législateur américain ajoute que les réparations ne peuvent être cédées qu'à des personnes encore en vie ; évitant ainsi de devoir, ultérieurement, donner des réparations aux amérindiens ou aux afro-américians. Cette loi n'empêche pas le négationnisme concernant l'internement, mais positionne clairement les États-Unis sur le sujet : officiellement, l'internement est condamné25.

En 2011, Edmund G. Brown Jr., Gouverneur de la Californie, dans un discours portant sur cette rescision est très clair :

That thousands of Japanese American citizens were wrongfully interned in American concentration camps without charge and without a fair hearing continues to trouble the conscience of this Nation. The internment of Japanese Americans should serve as a powerful reminder that in defending this Nation and its ideals, we must do so as faithfully in the courtrooms and the public squares of this country as upon the battlefields ${ }^{26}$.

Une fois de plus, l'accent est mis sur la citoyenneté des internés. Brown va encore plus loin en parlant de la loyauté de ces gens et de leur fidélité aux principes américains : "Japanese Americans remained steadfastly loyal to the United States throughout World War II. ... Although the National Government's fidelity to the ideals upon which it was founded lapsed during the war, the unshakeable faith of Japanese Americans in those same ideals did not " $^{27}$. Ce discours du Gouverneur fait écho à celui du Président Ford lors de la rescision de

\footnotetext{
${ }^{23}$ LyON Cherstin, « Japanese American Citizens League ", Densho Encyclopedia, 2013, disponible à l'adresse suivante: http://encyclopedia.densho.org/ (consultée le 18 décembre 2013).

${ }^{24}$ YANG Alice, "Redress Movement ", Densho Encyclopedia, 2013, disponible à l'adresse suivante : http:// encyclopedia.densho.org/Redress_movement/ (consultée le 18 décembre 2013).

${ }^{25}$ II convient de noter que l'internement n'occupe qu'une place mineure dans la mémoire américaine.

${ }^{26}$ BRown Edmund, «A Day of Remembrance: Japanese American Evacuation », Office of Governor Edmund G. Brown Jr., 2011, disponible à l'adresse suivante: http://gov.ca.gov/ (consultée le 17 décembre 2013) [souligné par l'auteure].

${ }^{27}$ lbid.
} 
l'ordre exécutif:

\begin{abstract}
We now know what we should have known then--not only was that evacuation wrong, but Japanese-Americans were and are loyal Americans. On the battlefield and at home, Japanese-Americans names like Hamada, Mitsumori, Marimoto, Noguchi, Yamasaki, Kido, Munemori and Miyamura - have been and continue to be written in our history for the sacrifices and the contributions they have made to the well-being and security of this, our common Nation ${ }^{28}$.
\end{abstract}

Ford, tout comme Brown, utilise la loyauté des Japonais américains pour créer un contraste encore plus fort entre les victimes et le Gouvernement, pour rendre ce qui leur est arrivé encore plus injuste. Dans ce genre de discours, les injustices commises contre les personnes de nationalité japonaise venues aux États-Unis pour se faire un peu d'argent, sans loyauté particulière au pays, ne sont également pas mentionnées. Afin de souligner le mal fait par le Gouvernement américain, ces discours mettent en avant l'atteinte portée à la liberté des Américains. De ce fait, les deux discours incorporent l'expérience des Japonais américains dans la mémoire collective américaine. Les internés deviennent des héros, au même titre que les Japonais américains ayant sacrifié leur vie dans l'armée. La liberté des internés a été sacrifiée de manière injuste. Les internés deviennent donc des citoyens à part entière mais également des Américains modèles.

Brown déclare le 19 février 2011 comme A Day of Remembrance : Japanese American Evacuation. II demande au peuple de la Californie de faire une solemn remembrance de la décision exécutive et de commémorer la réciscion de cet ordre ${ }^{29}$. Ce souvenir et cette commémoration ne sont cependant pas intrusifs : ce n'est pas un évènement annuel, mais simplement un discours pour rappeler aux citoyens un certain passé. Une forme de devoir de mémoire s'impose alors aux citoyens qui n'ont pas été victimes comme une contrainte morale. Ce devoir de mémoire prend une forme douce dans la mesure où il n'est pas gravé dans l'esprit des Américains comme étant un fait historique majeur. II ne s'impose pas régulièrement : de temps en temps, il est évoqué par une personnalité publique et des musées existent pour les personnes intéressées. Il est toutefois aisé pour un citoyen américain non japonais, de laisser le sable recouvrir les camps, petit à petit, jusqu'à ce qu'ils soient perdus dans le désert...

${ }^{28}$ FoRD Gerald, «President Gerald R. Ford's Proclamation 4417, Confirming the Termination of the Executive Order Authorizing Japanese-American Internment During World War II ", Selected Gerald R. Ford Presidential Speeches, 2000, disponible à l'adresse suivante : http://www.fordlibrarymuseum.gov/ (consultée le 18 décembre 2013) [souligné par l'auteure]. 